\title{
C-reactive protein haplotypes and dispositional optimism in obese and nonobese elderly subjects
}

\author{
Nathaly Rius-Ottenheim • Anton J. M. de Craen · Johanna M. Geleijnse • \\ P. Eline Slagboom • Daan Kromhout • Roos C. van der Mast • \\ Frans G. Zitman · Rudi G. Westendorp · Erik J. Giltay
}

Received: 15 June 2011/Revised: 4 August 2011/Accepted: 12 September 2011/Published online: 7 October 2011

(C) The Author(s) 2011. This article is published with open access at Springerlink.com

\begin{abstract}
Background Chronic low-grade inflammation, characterized by elevated plasma levels of C-reactive protein (CRP), has been inversely associated with dispositional optimism. Using a Mendelian randomization design, this study explores whether CRP haplotypes that determine CRP plasma levels are also associated with dispositional optimism.

Methods In a sample of 1,084 community-dwelling subjects (aged 60-85 years) from three cohort studies (Arnhem Elderly Study, $n=426$; Leiden Longevity Study, $n=355$; Zutphen Elderly Study, $n=303$ ), six CRP polymorphisms (rs2808628, rs2808630, rs1205, rs1800947, rs1417938, and rs3091244) coding for five common haplotypes were genotyped. The association of CRP haplotypes with CRP plasma levels and dispositional optimism was analyzed using
\end{abstract}

Responsible Editor: Andras Falus.

N. Rius-Ottenheim $(\bowtie) \cdot$ R. C. van der Mast .

F. G. Zitman · E. J. Giltay

Department of Psychiatry, Leiden University Medical Centre,

P.O. Box 9600, 2300 RC Leiden, The Netherlands

e-mail: N.Rius_Ottenheim@lumc.nl

A. J. M. de Craen · R. G. Westendorp

Department of Gerontology and Geriatrics, Leiden University

Medical Centre, Leiden, The Netherlands

A. J. M. de Craen · P. E. Slagboom - R. G. Westendorp Netherlands Consortium for Healthy Ageing,

Leiden, The Netherlands

J. M. Geleijnse · D. Kromhout

Division of Human Nutrition, Wageningen University,

Wageningen, The Netherlands

P. E. Slagboom

Department of Molecular Epidemiology, Leiden University

Medical Centre, Leiden, The Netherlands multivariable linear regression models. Subanalyses were stratified by body mass index (BMI $\geq 25 \mathrm{~kg} / \mathrm{m}^{2}$ ).

Results CRP haplotypes determined CRP plasma levels (adjusted $\beta=0.094, p<0.001$ ). In the whole group, no association was found between CRP haplotypes and dispositional optimism scores (adjusted $\beta=-0.02, p=$ 0.45). In BMI strata, CRP haplotypes were associated with increasing levels of plasma CRP levels (adjusted $\beta=$ $0.112 ; p=0.002)$ and lower dispositional optimism levels (adjusted $\beta=-0.068 ; p=0.03$ ) in the obese group only. Conclusions These results suggest that genetically increased CRP levels are involved in low dispositional optimism, but only in case of obesity.

Keywords Obesity - CRP - Dispositional optimism . Inflammation · Genotypes · Haplotypes

\section{Introduction}

Chronic low-grade inflammation is proposed as one of the underlying mechanisms through which psychological factors influence a range of health outcomes [1], including mortality risk [2,3]. Although most studies addressing the association between inflammation and mood states have focused on the link with negative affect [4-8], there is increasing evidence for an association between inflammation and positive affect [9-12]. These studies reported an inverse association of positive affective states with proinflammatory acute-phase proteins and cytokines, such as interleukin-6 (IL-6) $[9,10]$ and plasma fibrinogen $[11,12]$.

Inflammation is also associated with the positive affective trait dispositional optimism, which is defined as generalized expectancy of positive outcomes for the future [13]. Dispositional optimism has been associated with 
better outcomes in mental and physical health [14-17]. These benefits may result from the more effective coping used by optimists and its effects on stress resilience. It is suggested that greater stress resilience may lead to downregulation of the inflammatory response by counteracting the neuroendocrine reaction to stressful events [18]. Furthermore, it is also possible that chronic low-grade inflammation lowers the level of dispositional optimism. In a recent cross-sectional study among middle-aged healthy adults $(n=6,814)$, circulating levels of C-reactive protein (CRP) [11], a nonspecific systemic inflammatory acutephase protein, were inversely associated with higher dispositional optimism scores. However, the cross-sectional nature of the reported association between inflammation and optimism does not allow drawing conclusions about causal pathways. It remains unclear whether low levels of optimism are secondary to inflammation, or low inflammatory markers are secondary to optimism, or whether a third factor is involved in the putative association.

It is well known that (abdominal) obesity is strongly related to inflammation and may therefore act as a potential moderator of the association between inflammation and dispositional optimism. Visceral fat is an important determinant of moderately increased CRP levels in both men and women [19]. Furthermore, weight changes have been correlated to changes in CRP levels [20, 21]. In an earlier study, a gene-environment interaction was reported for the effect of body mass index (BMI) on CRP plasma levels [22]. In obese subjects, a stronger association was found between the CRP single-nucleotide polymorphism (SNP) rs1205 and plasma CRP levels than in lean subjects, especially in men.

Mendelian randomization has been used to investigate biological relationships by inferring causality when unobserved confounding is likely [23-25]. This approach is based on the random allocation of alleles at the moment of gamete formation and can be considered as a natural form of randomized controlled trial [26]. Previous studies using Mendelian randomization have shown that CRP transcription and plasma concentrations depend on genetic variation at single-nucleotide polymorphisms (SNPs): rs2808628, rs2808630, rs1205, rs1800947, rs1417938, and rs3091244 [27-33]. Furthermore, a recent Mendelian randomization study showed that the observed association between CRP and BMI is most likely driven by BMI, with CRP being raised due to obesity [34]. Regarding affective states, the relationship with CRP genetic variation has only been studied for depressive symptoms, with negative findings in two previous cross-sectional studies among 868 middleaged subjects and 3,700 community-dwelling elderly subjects $[35,36]$. The relationship between CRP genotype and the positive affective trait dispositional optimism remains largely unexplored.
The present study investigates the associations between CRP haplotypes and dispositional optimism in three different samples of older subjects. We hypothesized that CRP haplotypes associated with higher levels of CRP in plasma may also contribute to lower levels of dispositional optimism. Since obesity is strongly related to inflammation and may modulate the association between CRP haplotypes and CRP plasma levels [22], we also investigated the effect of BMI on the association between CRP haplotypes and dispositional optimism by repeating the analyses stratifying by BMI.

\section{Methods}

\section{Study sample}

The present study uses data from participants of the Arnhem Elderly Study (AES), the Leiden Longevity Study (LLS), and the Zutphen Elderly Study (ZES). The study design and population characteristics of these three samples have been described in detail elsewhere [37-39]. The AES is a population-based study among 685 noninstitutionalized adults (aged 65-84 years) who agreed to undergo venipuncture to investigate lifestyle and health characteristics [40]. In the LLS, 420 families with longlived Caucasian siblings, together with their offspring and the offspring's partners, were recruited to study predictors and mechanisms of longevity. Families were eligible for the study if at least two long-lived, full siblings were alive, where men were considered to be long-lived if they were 89 years or older and women if they were 91 years or older [38]. The ZES is a longitudinal investigation of risk factors for chronic diseases among Caucasian men (aged 65-84 years), of whom 560 participated in 1990 [37]. Participants of the three samples were included in subsequent analysis when having complete data on CRP plasma concentrations, dispositional optimism scores, and at least four of the six CRP SNPs. In a number of subjects DNA was not available. This selection criterion resulted in a final study sample of 1,084 participants: 426 Caucasian subjects aged 65-85 years from the AES, 355 Caucasian subjects aged 60-84 years from the LLS, and 303 Caucasian subjects aged 70-89 years from the ZES. All studies were conducted in compliance with the principles of the Helsinki Accord and were approved by the local ethics committees. Standard informed consent was obtained from all participants.

\section{Plasma CRP measurement and CRP SNP genotyping}

Blood samples were drawn by standard venipuncture techniques. Plasma CRP was assayed with a highly 
sensitive enzyme-linked immunosorbent assay procedure in the AES and with automated enzymatic colorimetric methods using a Modular P analyzer (Roche, Switzerland) in the LLS. CRP plasma levels were not measured in the ZES, and therefore CRP levels were available in $781(72 \%)$ of 1,084 subjects.

Genomic DNA was isolated from the blood samples according to standard procedures. SNPs at the CRP gene were determined together with the frequency of six wellcharacterized CRP polymorphisms shown to be related to plasma CRP levels (Fig. 1). I-plex assays were assigned using Assay designer software (Sequenom). Genotyping was performed using the MassArray platform according to the manufacturer's protocols (Sequenom, San Diego, CA). After polymerase chain reaction (PCR) on $2.5 \mathrm{ng}$ DNA, a primer extension reaction was performed to introduce mass differences between alleles. After removing salts by adding a resin, $\sim 15 \mathrm{~nL}$ of the product was spotted onto a target chip with 384 patches containing matrix. Mass differences were detected using a Bruker Autoflex matrix-assisted laser desorption/ionization time-of-flight (MALDI-TOF) mass spectrometer, and genotypes were assigned in real time using Typer 3.1 software (Sequenom). Genotype frequencies of these SNPs showed no significant deviations from Hardy-Weinberg equilibrium (all $p$ values $>0.05$, except for SNP rs3091244 in the ZES with $p=0.03$ ).

The haplotype frequencies were generated using SNPHAP (Clayton, 2002; version 1.3; available online at http:// www-gene.cimr.cam.ac.uk/clayton/software/) with an estimation-maximization algorithm. Six SNPs (i.e., rs2808628, rs2808630, rs1205, rs1800947, rs1417938, and rs3091244) were used to generate five common haplotypes that determine about $10 \%$ of the interindividual variability in CRP levels in the absence of infection [27, 31]. Haplotypes with prevalence larger than 3\% were selected for further comparison and named haplotype 1 , haplotype 2 , haplotype 3 , haplotype 4, and haplotype 5. Haplotypes were placed in numerical order of their known association with increasing plasma CRP levels [28, 33].

\section{Dispositional optimism}

Dispositional optimism was assessed using the Dutch Scale for Subjective Well-being for Older persons (SSWO) in 426 participants in the AES [41], using the Life Orientation Test-Revised (LOT-R) in 355 participants in the LLS [13], and using the four-item optimism questionnaire (4Q) in 303 participants in the ZES [16]. The SSWO consists of 30 items rated on three-point scales divided into five subscales, including an optimism subscale composed of seven items [15]. Within the seven items yielding an optimism score, one item required reversed coding. A typical item was: "I still expect much from life." The total score ranges from 0 to 14 points $[15,40,41]$. The LOT-R consists of 10 items rated on a five-point Likert scale, with 6 items yielding an optimism score and 4 filler items [13]. Within the six score items, three negatively stated items required reversed coding. A typical questionnaire item was: "Overall, I expect more good things to happen to me than bad things." The total LOT-R score ranges from 0 to 24 points. The 4Q [16] consists of four statements rated on a three-point Likert scale, such as "I am still full of plans." Within the four score items, two negatively stated items required reversed coding. The additional answer category "do not know" was coded as the midpoint (score 1). The total score ranges from 0 to 8 points. For computation of optimism scores, one missing item per subject was allowed, being subsequently imputed as the mean of the

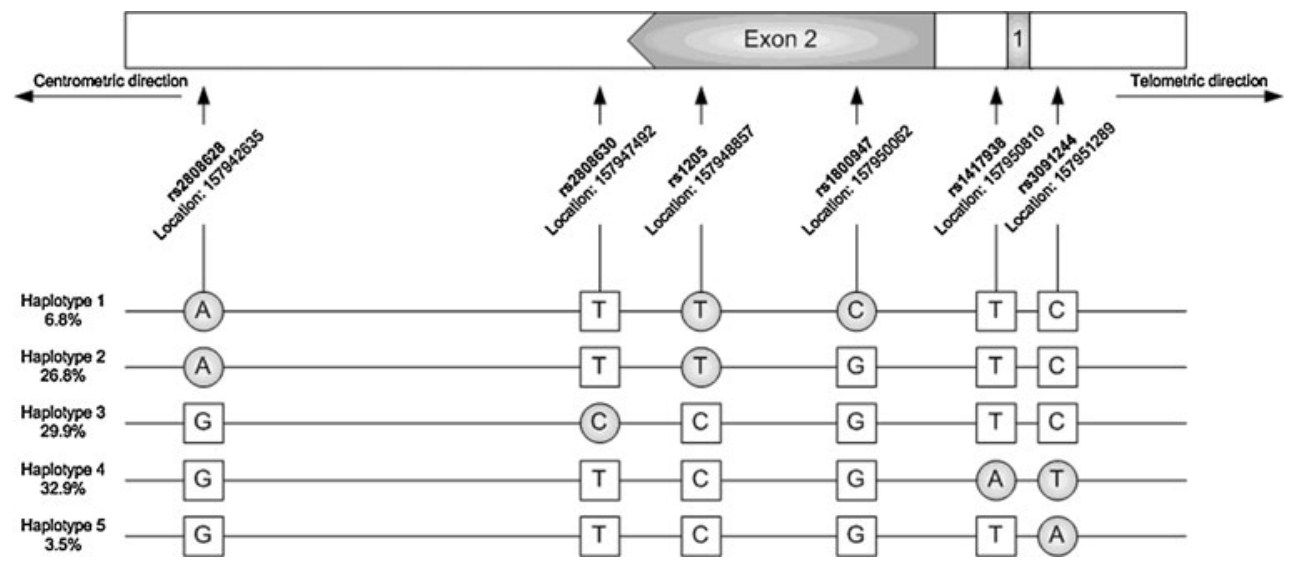

Fig. 1 Schematic overview of the structure of the C-reactive protein gene, consisting of two exons separated by a single intron. The orientation of the gene is marked by arrows, with the gene transcribed from left to right. Arrows mark the approximate location of the six single-nucleotide polymorphisms that are in close linkage disequilibrium. The five most frequent haplotypes are presented, similar to those found in populations of Northern and Western European ancestry. Note that the percentages do not sum to $100 \%$ because infrequent haplotypes were not taken into account 
remaining seven, five, or three items for the SSWO, the LOT-R, and the 4Q, respectively. Higher scores on all three optimism scales were indicative of higher optimism levels. Similar indices of moderate internal consistency were found for the three optimism scales (with Cronbach's $\alpha$ of: SSWO 0.76, LOT-R 0.67, and 4Q 0.65).

\section{Other variables}

Sociodemographic and lifestyle characteristics were obtained from all participants, including age and gender. Marital status was dichotomized as being married or not. Smoking status was classified as no smoking, former smoking, and current smoking. Alcohol use was dichotomized as current alcohol consumption or no alcohol consumption. BMI was calculated from the measured weight and height, and two strata were formed according to the cutoff of $25 \mathrm{~kg} / \mathrm{m}^{2}$. History of cancer and history of cardiovascular disease (i.e., heart failure, myocardial infarction, or stroke) were also dichotomized.

\section{Statistical analysis}

Sociodemographic and lifestyle characteristics were summarized using descriptive statistics. Differences across the three studies were analyzed using chi-square tests and oneway analysis of variance (ANOVA), when appropriate. Results are presented as number and percentage for categorical variables, mean and standard deviation (SD) for continuous normally distributed variables, and median with interquartile range for continuous skewed variables. Logarithmic transformation was applied to normalize distributions of the positively skewed CRP values, and subsequent analyses were conducted on these transformed values. Back-transformed geometric mean values are presented in all tables. To enable comparison of the optimism scores obtained by the three different scales, $z$-scores (i.e., standardized scores) were used.

First, one-way ANOVAs were used to assess the associations between the alleles of the six SNPs and dispositional optimism within each sample. Given the low frequency of the minor allele of SNP rs 1800947, the alleles $\mathrm{GC} / \mathrm{CC}$ were pooled into one group.

Second, univariate ANOVAs were used to study the association between CRP haplotypes and circulating CRP. The same analyses were used to investigate the relationship between CRP haplotypes and dispositional optimism. In each analysis, the association was subsequently tested adjusting for potential confounders, including age, gender, marital status, smoking status, alcohol use, history of cancer or cardiovascular disease, and cohort (when appropriate). Finally, to assess the effect modification of obesity on the association between CRP haplotypes and CRP plasma levels or dispositional optimism, we stratified according to BMI group (i.e., group 1 had BMI $<25 \mathrm{~kg} / \mathrm{m}^{2}$, group 2 had $\mathrm{BMI} \geq 25 \mathrm{~kg} / \mathrm{m}^{2}$ ) [42]. Linear regression analyses (weighted for haplotype probability) were used to test for linear trend over the haplotypes ordered according to increasing CRP level. Finally, to unveil potential differences between men and women, the same analyses were repeated testing the interaction with gender.

All $p$ values are two-tailed and considered statistically significant at the level of $p<0.05$. Data analyses were performed using SPSS version 17.0 (Chicago, IL) for Windows.

\section{Results}

Sociodemographic characteristics

Sociodemographic and lifestyle characteristics are summarized in Table 1. The mean age of the study sample was 71 (SD 7) years. The percentage of male participants was around $45 \%$ in the AES and the LLS, whereas the ZES study was solely composed of men. Participants of the AES more frequently reported a history of cardiovascular disease. Finally, median levels of circulating CRP were similar across the two studies with available data (i.e., AES and LLS), and a positive association between serum CRP levels and BMI (adjusted $\beta=0.35 ; p<0.001$ ) was found.

\section{CRP genotype and CRP levels}

Figure 1 presents the five haplotypes used in this study, with prevalence rates highly consistent with those found in populations of Northern/Western European origin [33, 43, 44]. Genetic variation at these chromosomal regions is represented by so-called tag SNPs (also depicted in Fig. 1). As expected, results from the linear regression analysis showed that CRP haplotypes were associated with increasing CRP plasma levels ( $\beta=0.088 ; p=0.001)$ in the combined study sample. In the multivariable model, this association persisted ( $\beta=0.094 ; p<0.001)$. Largely similar results were found when studying the association within samples. After stratification by BMI, the adjusted association between CRP haplotypes and increasing levels of circulating CRP only persisted in the obese group ( $\beta=0.112 ; p=0.002)$, but disappeared in the nonobese group $(\beta=0.069 ; p=0.08)$ (Fig. 2). The difference in strength of the associations across the obesity groups was significant after adjustment for marital status, age, gender, alcohol, smoking habits, cardiovascular disease, cancer, and cohort.

CRP and dispositional optimism

There was an inverse association between serum CRP levels and dispositional optimism ( $\beta=-0.053 ; p=0.03$ ). 
Table 1 Sociodemographic and clinical characteristics of the 1,084 participants

\begin{tabular}{lllll}
\hline & Total $(n=1,084)$ & ZES $(n=303)$ & AES $(n=426)$ & LLS $(n=355)$ \\
\hline Age $($ years $)$, mean \pm SD & $70.7 \pm 6.6$ & $70.6 \pm 4.3$ & $74.3 \pm 5.7$ & $66.2 \pm 4.5$ \\
Male, $n(\%)$ & $698(64.4)$ & $303(100)$ & $205(47.9)$ & $192(54.1)$ \\
Married, $n(\%)$ & $864(80.4)$ & $265(89.8)$ & $260(60.7)$ & $339(95.8)$ \\
Current smoker, $n(\%)$ & $220(20.3)$ & $80(26.4)$ & $97(22.7)$ & $44(12.5)$ \\
Alcohol use, $n(\%)$ & $760(70.1)$ & $230(78.5)$ & $266(70.2)$ & $265(75.3)$ \\
Cardiovascular disease, $n(\%)$ & $161(15.4)$ & $35(11.6)$ & $100(23.6)$ & $26(8.2)$ \\
Cancer, $n(\%)$ & $61(5.8)$ & $17(5.6)$ & $18(4.2)$ & $26(8.3)$ \\
Body mass index, mean \pm SD $\left(\mathrm{kg} / \mathrm{m}^{2}\right)$ & $25.8 \pm 3.7$ & $25.6 \pm 2.8$ & $25.9 \pm 3.9$ & $25.8 \pm 3.5$ \\
Body mass index $\geq 25 \mathrm{~kg} / \mathrm{m}^{2}$ & $595(54.9 \%)$ & $172(56.8 \%)$ & $228(53.5 \%)$ & $195(54.9 \%)$ \\
C-reactive protein, median $(\mathrm{p} 25 ; \mathrm{p} 75)$ & $1.80(0.90 ; 3.60)$ & - & $2.30(1.18 ; 4.10)$ & $1.40(0.74 ; 2.86)$
\end{tabular}

ZES Zutphen Elderly Study, AES Arnhem Elderly Study, LLS Leiden Longevity Study, SD standard deviation

Fig. 2 Mean standard scores (with error bars representing standard errors) for C-reactive protein (CRP) levels according to CRP gene haplotypes. The five common haplotypes were constructed from rs 2808628 , rs2808630, rs1205, rs1800947, rs1417938, and rs3091244. The size of each square is proportional to the number of measurements. Scores are adjusted for age, gender (two categories), smoking status (three categories), alcohol use (two categories), prevalent cancer (two categories), prevalent cardiovascular disease (two categories), and cohort (three categories). $p$-Values are for linear trend (by weighted regression analysis) over increasing haplotypes
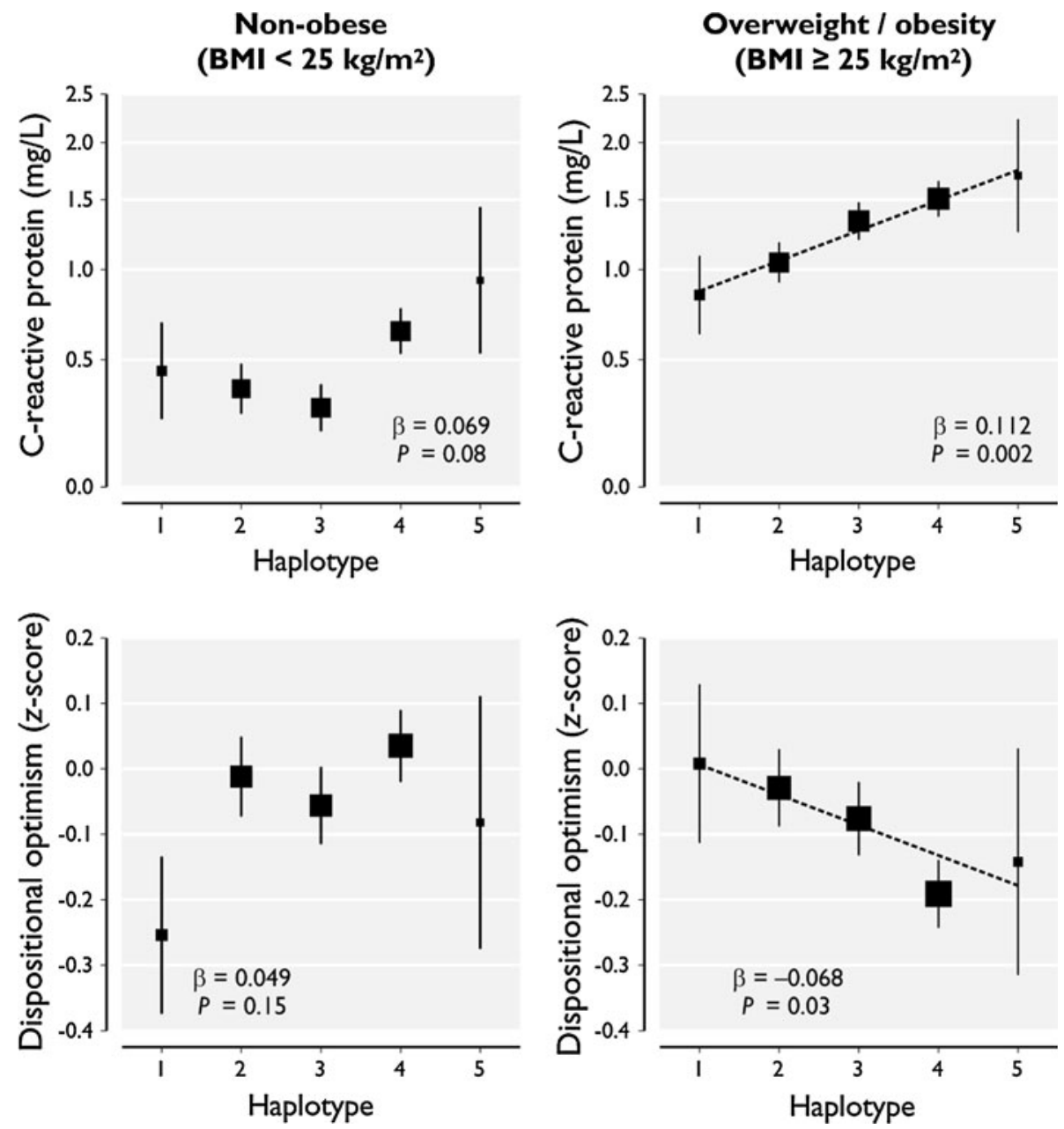

However, this association disappeared after adjustment for the following confounders: age, gender, marital status, smoking status, alcohol use, and history of cancer or cardiovascular disease $(\beta=-0.006 ; p=0.83)$.
Table 2 presents results on the association between CRP haplotypes and dispositional optimism. No significant overall association was found between CRP haplotypes and levels of dispositional optimism in the combined sample. 
However, a marginally significant association was found in the AES after adjusting for potential confounders. After stratification by BMI, an association was found between CRP haplotypes and dispositional optimism ( $z$-scores) in the obese group $(n=595 ; \beta=-0.068 ; p=0.03)$, but not in the nonobese group $(n=489 ; \beta=0.049 ; \quad p=0.15)$ (Fig. 2). The difference in strength of the associations across the obesity groups was significant after adjustment for marital status, age, gender, alcohol, smoking habits, cardiovascular disease, cancer, and cohort ( $p$ for interaction term haplotype $\times$ optimism was 0.04). Associations between CRP haplotypes and dispositional optimism in obese subjects were of similar strength in obese men $(\beta=$ $-0.070)$ and obese women $(\beta=-0.067)$, and the interaction term with gender was not statistically significant.

\section{Discussion}

The present study aimed to determine whether specific haplotypes of the CRP gene known to affect gene transcription and circulating levels of CRP [27-33, 43, 44] were also associated with dispositional optimism. The association between C-reactive protein (CRP) and low dispositional optimism was tested using a Mendelian randomization design. There was an association between CRP genotype and circulating serum CRP levels. However, no association was found between CRP genotype and low optimism, implying that a causal association is unlikely. The lack of relationship suggests that reverse causation, bias, or confounding generated the link between high CRP plasma levels and low optimism. Our data indicated that BMI was a likely confounder of the relationship, as subjects with higher BMI displayed higher CRP levels and lower optimism. Since previous studies had reported a gene-environment interaction for the relationship between BMI and CRP levels [22], we performed extra analyses stratified by BMI. BMI modulated the association between CRP haplotypes and dispositional optimism, as an inverse association was only found in the obese group.

Although our findings are interesting and merit further investigation, some study limitations need to be addressed. First, three different instruments were used to measure dispositional optimism. Second, it should be noted that about $25 \%$ of the LLS were sibling pairs, and therefore not genetically independent. Third, although we included three cohorts in the analyses, the total number of 1,084 participants is relatively small for a genetic association study. Finally, we restricted our analysis to Caucasian European subjects, which limits the generalization of our findings to non-Caucasian populations. Given the evidence that circulating levels of CRP are higher in African Americans than European Caucasians [27, 28], further research in nonCaucasian populations may be needed.

Table 2 Associations between common C-reactive protein (CRP) haplotypes, plasma CRP levels, and dispositional optimism in 1,084 participants

\begin{tabular}{|c|c|c|c|c|c|c|c|c|c|}
\hline \multirow[t]{3}{*}{ Haplotype } & \multirow[t]{3}{*}{ Genotype combination } & \multicolumn{8}{|c|}{ Dispositional optimism } \\
\hline & & \multicolumn{2}{|c|}{ AES } & \multicolumn{2}{|l|}{ LLS } & \multicolumn{2}{|l|}{ ZES } & \multicolumn{2}{|c|}{ Overall } \\
\hline & & $n$ & Mean (SE) & $n$ & Mean (SE) & $n$ & Mean (SE) & $n$ & Mean (SE) \\
\hline \multicolumn{10}{|l|}{ Crude } \\
\hline 1 & АТTCTC & 28 & $-0.14(0.13)$ & 27 & $-0.03(0.13)$ & 18 & $-0.16(0.15)$ & 47 & $-0.10(0.08)$ \\
\hline 2 & ATTGTC & 115 & $0.19(0.07)$ & 90 & $-0.07(0.07)$ & 79 & $-0.25(0.07)$ & 177 & $-0.01(0.04)$ \\
\hline 3 & GCCGTC & 131 & $-0.06(0.06)$ & 103 & $-0.07(0.07)$ & 79 & $-0.11(0.07)$ & 203 & $-0.07(0.04)$ \\
\hline 4 & GTCGAT & 126 & $-0.08(0.06)$ & 115 & $-0.01(0.06)$ & 108 & $-0.14(0.06)$ & 216 & $-0.07(0.04)$ \\
\hline \multirow[t]{2}{*}{5} & GTCGTA & 17 & $-0.10(0.17)$ & 9 & $-0.14(0.23)$ & 12 & $0.05(0.26)$ & 22 & $-0.08(0.12)$ \\
\hline & & \multicolumn{2}{|c|}{$\beta=-0.066 ; p=0.057$} & \multicolumn{2}{|c|}{$\beta=0.012 ; p=0.76$} & \multicolumn{2}{|c|}{$\beta=0.045 ; p=0.27$} & & $p=0.55$ \\
\hline \multicolumn{10}{|l|}{ Adjusted* } \\
\hline 1 & ATTCTC & 24 & $-0.18(0.14)$ & 23 & $-0.09(0.15)$ & 17 & $-0.09(0.16)$ & 64 & $-0.12(0.09)$ \\
\hline 2 & ATTGTC & 100 & $0.16(0.07)$ & 79 & $-0.06(0.08)$ & 77 & $-0.23(0.07)$ & 254 & $-0.02(0.04)$ \\
\hline 3 & GCCGTC & 113 & $-0.04(0.06)$ & 93 & $-0.08(0.07)$ & 73 & $-0.11(0.07)$ & 276 & $-0.07(0.04)$ \\
\hline 4 & GTCGAT & 115 & $-0.12(0.06)$ & 102 & $-0.02(0.07)$ & 104 & $-0.12(0.06)$ & 320 & $-0.09(0.04)$ \\
\hline \multirow[t]{2}{*}{5} & GTCGTA & 14 & $-0.20(0.18)$ & 8 & $-0.03(0.25)$ & 12 & $0.07(0.25)$ & 33 & $-0.12(0.13)$ \\
\hline & & \multicolumn{2}{|c|}{$\beta=-0.072 ; p=0.047$} & \multicolumn{2}{|c|}{$\beta=0.019 ; p=0.63$} & \multicolumn{2}{|c|}{$\beta=0.039 ; p=0.35$} & & $p=0.45$ \\
\hline
\end{tabular}

Five common haplotypes were constructed from rs2808628, rs2808630, rs1205, rs1800947, rs1417938, and rs3091244. Weighted linear regression analysis was used to test for linear trend over the five haplotypes

* Geometric estimated means adjusted for age, gender, marital status, smoking status, alcohol use, history of cancer or cardiovascular disease, and cohort (when appropriate) with standard errors (SE) are presented for dispositional optimism 
Our results are in line with the findings of the crosssectional Multi-Ethnic Study of Atherosclerosis (MESA) among 6,184 middle-aged adults, which showed that higher LOT-R scores were inversely associated with serum CRP levels [11]. Similar to the present study, this association attenuated after adjustment for sociodemographic and lifestyle-related factors, including BMI. Similarly, our results concur with findings from other cross-sectional studies investigating positive affect, a construct closely related to optimism [10, 12, 45]. We have extended their findings by investigating the role of allelic variation at the CRP gene and by exploring the modifying effect of obesity on the association between the CRP gene and both CRP levels and dispositional optimism. We found that the association between CRP haplotypes and CRP levels was stronger in subjects with obesity. This confirms findings of another study (among 2,296 older subjects) reporting that the association between the minor allele of the CRP SNP rs1205 and higher CRP levels was also stronger in subjects with higher BMI [22]. Likewise, in another study, CRP was related to poorer cognition in women with $\mathrm{BMI}$ higher than $25 \mathrm{~kg} / \mathrm{m}^{2}$, but not in leaner women [42]. We found that CRP haplotypes were associated with dispositional optimism when BMI was higher than $25 \mathrm{~kg} / \mathrm{m}^{2}$; this suggests that high CRP levels are associated with lower optimism, but only in obese subjects.

It is well known that the volume and distribution of adipose tissue are critical in the production of CRPinducing factors [19, 34, 46]. One of these factors is IL-6, a cytokine produced by adipose tissue that regulates hepatic synthesis of CRP [47]. Since this proinflammatory cytokine is also associated with positive affect [10], it is worthwhile to study both IL-6 and CRP in relation to optimism in obese and nonobese subjects, as the interaction of many other proinflammatory proteins [e.g., tumor necrosis factor$\alpha(\mathrm{TNF}-\alpha)$ ] with obesity is complex.

Although the Mendelian randomization design makes it less likely, we cannot exclude inverse causation as a possible explanation for our findings, i.e., that dispositional optimism may affect systemic inflammation rather than vice versa. Dispositional optimism is associated with health-promoting behaviors such as nonsmoking, increased physical activity, moderate alcohol consumption, and more fruits, vegetables, and whole grains in the diet [48]. Therefore, the association between dispositional optimism and inflammation levels might be moderated through longstanding changes in health behaviors and lifestyle, such as healthy diets and weight reduction. Dispositional optimism is associated with greater resilience to stress, which in turn may reduce the affective reaction to a stressful event. Otherwise, poor coping strategies result in continued and enhanced activation of the hypothalamic-pituitary-adrenal (HPA) axis, inducing a cascade of neuroendocrine and hormonal changes [49]. Thus, chronic dysregulation of the HPA axis may also contribute to inflammatory processes. By favoring more benign appraisals of stress, optimism may lead to downregulation of the neuroendocrine system and, thereby, to decreased levels of inflammatory cytokines and acute-phase proteins [18]. Nevertheless, under conditions of acute biological or psychological stressors, optimism may help to boost inflammatory processes favoring a more rapid and successful response to the stressor. For example, in a study among 18 healthy older adults, those with higher levels of optimism showed greater in vitro IL-2 and interferon- $\gamma$ responses to viral stimulation [50].

In summary, our study indicates that allelic variation at the CRP gene is related to CRP levels and dispositional optimism, and that the strength of this association is modulated by BMI. Further prospective studies are necessary to confirm our findings. Such studies could investigate the role of BMI in the association between inflammation and affective states also incorporating other markers of inflammation, such as IL-6 or TNF- $\alpha$.

Acknowledgments The Arnhem Elderly Study was supported by the Dutch Ministry of Health, Welfare, and Sport. The Leiden Longevity Study was funded by the Innovation Oriented Research Program on Genomics (SenterNovem; IGE01014 and IGE5007), the Centre for Medical Systems Biology, the Netherlands Genomics Initiative/Netherlands Organization for Scientific Research (05040202 and 050-060-810), and the European Union-funded Network of Excellence Lifespan (FP6 036894). The Zutphen Elderly Study was supported by the Netherlands Prevention Foundation, The Hague. Part of this study was funded by a grant from Brain Foundation of the Netherlands [Hersenstichting Nederland, grant number 15F07 (2) 24].

Conflict of interest No disclosures to report.

Open Access This article is distributed under the terms of the Creative Commons Attribution Noncommercial License which permits any noncommercial use, distribution, and reproduction in any medium, provided the original author(s) and source are credited.

\section{References}

1. Ranjit N, Diez-Roux A, Shea S, Cushman M, Seeman T, Jackson $\mathrm{S}$, et al. Psychosocial factors and inflammation in the multi-ethnic study of atherosclerosis. Arch Intern Med. 2007;167(2):174-81.

2. Steptoe A, Hamer M, Chida Y. The effects of acute psychological stress on circulating inflammatory factors in humans: a review and meta-analysis. Brain Behav Immun. 2007;21(7):901-12.

3. Carney R, Freedland K, Miller G, Jaffe A. Depression as a risk factor for cardiac mortality and morbidity: a review of potential mechanisms. J Psychosom Res. 2002;53(4):897-902.

4. Bremmer M, Beekman A, Deeg D, Penninx B, Dik M, Hack C, et al. Inflammatory markers in late-life depression: results from a population-based study. J Affect Disord. 2008;106(3):249-55.

5. Danner M, Kasl S, Abramson J, Vaccarino V. Association between depression and elevated C-reactive protein. Psychosom Med. 2003;65(3):347-56. 
6. Ford D, Erlinger T. Depression and C-reactive protein in US adults: data from the Third National Health and Nutrition Examination Survey. Arch Intern Med. 2004;164(9):1010-4.

7. Kop W, Gottdiener J, Tangen C, Fried L, McBurnie M, Walston $\mathrm{J}$, et al. Inflammation and coagulation factors in persons $>65$ years of age with symptoms of depression but without evidence of myocardial ischemia. Am J Cardiol. 2002;89(4): 419-24.

8. Penninx B, Kritchevsky S, Yaffe K, Newman A, Simonsick E, Rubin $\mathrm{S}$, et al. Inflammatory markers and depressed mood in older persons: results from the health, aging and body composition study. Biol Psychiatry. 2003;54(5):566-72.

9. Friedman E, Hayney M, Love G, Singer B, Ryff C. Plasma interleukin-6 and soluble IL-6 receptors are associated with psychological well-being in aging women. Health Psychol. 2007;26(3):305-13.

10. Janicki-Deverts D, Cohen S, Doyle W, Turner R, Treanor J. Infection-induced proinflammatory cytokines are associated with decreases in positive affect, but not increases in negative affect. Brain Behav Immun. 2007;21(3):301-7.

11. Roy B, Diez-Roux A, Seeman T, Ranjit N, Shea S, Cushman M. Association of optimism and pessimism with inflammation and hemostasis in the Multi-Ethnic Study of Atherosclerosis (MESA). Psychosom Med. 2010;72(2):134-40.

12. Steptoe A, Wardle J, Marmot M. Positive affect and healthrelated neuroendocrine, cardiovascular, and inflammatory processes. Proc Natl Acad Sci USA. 2005;102(18):6508-12.

13. Scheier M, Carver C, Bridges M. Distinguishing optimism from neuroticism (and trait anxiety, self-mastery, and self-esteem): a reevaluation of the life orientation test. J Pers Soc Psychol. 1994;67(6):1063-78.

14. Carver C, Scheier M, Segerstrom S. Optimism. Clin Psychol Rev. 2010;30(7):879-89.

15. Giltay E, Geleijnse J, Zitman F, Hoekstra T, Schouten E. Dispositional optimism and all-cause and cardiovascular mortality in a prospective cohort of elderly dutch men and women. Arch Gen Psychiatry. 2004;61(11):1126-35.

16. Giltay E, Kamphuis M, Kalmijn S, Zitman F, Kromhout D. Dispositional optimism and the risk of cardiovascular death: the Zutphen Elderly Study. Arch Intern Med. 2006;166(4):431-6.

17. Tindle H, Chang Y, Kuller L, Manson J, Robinson J, Rosal M, et al. Optimism, cynical hostility, and incident coronary heart disease and mortality in the women's health initiative. Circulation. 2009; 120(8):656-62.

18. Brydon L, Walker C, Wawrzyniak A, Chart H, Steptoe A. Dispositional optimism and stress-induced changes in immunity and negative mood. Brain Behav Immun. 2009;23(6):810-6.

19. Visser M, Bouter L, McQuillan G, Wener M, Harris T. Elevated C-reactive protein levels in overweight and obese adults. JAMA. 1999;282(22):2131-5.

20. Festa A, D’Agostino RJ, Williams K, Karter A, Mayer-Davis E, Tracy $\mathrm{R}$, et al. The relation of body fat mass and distribution to markers of chronic inflammation. Int J Obes Relat Metab Disord. 2001;25(10):1407-15.

21. Tchernof A, Nolan A, Sites C, Ades P, Poehlman E. Weight loss reduces C-reactive protein levels in obese postmenopausal women. Circulation. 2002;105(5):564-9.

22. Eiriksdottir G, Smith A, Aspelund T, Hafsteinsdottir S, Olafsdottir E, Launer L, et al. The interaction of adiposity with the CRP gene affects CRP levels: age, gene/environment susceptibilty-Reykjavik study. Int J Obes (Lond). 2009;33(2):267-72.

23. Lawlor D, Harbord R, Sterne J, Timpson N, Smith DG. Mendelian randomization: using genes as instruments for making causal inferences in epidemiology. Stat Med. 2008;27(8):1133-63.

24. Smith G, Ebrahim S. Mendelian randomization: prospects, potentials, and limitations. Int J Epidemiol. 2004;33(1):30-42.
25. Bochud M, Rousson V. Usefulness of Mendelian randomization in observational epidemiology. Int J Environ Res Public Health. 2010;7(3):711-28.

26. Sheehan N, Didelez V, Burton P, Tobin M. Mendelian randomisation and causal inference in observational epidemiology. PLoS Med. 2008;5(8):e177.

27. Lange L, Carlson C, Hindorff L, Lange E, Walston J, Durda J, et al. Association of polymorphisms in the CRP gene with circulating C-reactive protein levels and cardiovascular events. JAMA. 2006;296(22):2703-11.

28. Carlson C, Aldred S, Lee P, Tracy R, Schwartz S, Rieder M, et al. Polymorphisms within the C-reactive protein (CRP) promoter region are associated with plasma CRP levels. Am J Hum Genet. 2005;77(1):64-77.

29. Crawford D, Sanders C, Qin X, Smith J, Shephard C, Wong M, et al. Genetic variation is associated with $\mathrm{C}$-reactive protein levels in the Third National Health and Nutrition Examination Survey. Circulation. 2006;114(23):2458-65.

30. Szalai A, McCrory M, Cooper G, Wu J, Kimberly R. Association between baseline levels of C-reactive protein (CRP) and a dinucleotide repeat polymorphism in the intron of the CRP gene. Genes Immun. 2002;3(1):14-9.

31. Hage F, Szalai A. C-reactive protein gene polymorphisms, C-reactive protein blood levels, and cardiovascular disease risk. J Am Coll Cardiol. 2007;50(12):1115-22.

32. Kathiresan S, Larson M, Vasan R, Guo C, Gona P, Keaney JJ, et al. Contribution of clinical correlates and $13 \mathrm{C}$-reactive protein gene polymorphisms to interindividual variability in serum C-reactive protein level. Circulation. 2006;113(11):1415-23.

33. Bos S, Suchiman H, Kloppenburg M, Houwing-Duistermaat J, le Graverand M, Seymour A, et al. Allelic variation at the C-reactive protein gene associates to both hand osteoarthritis severity and serum high sensitive C-reactive protein levels in the GARP study. Ann Rheum Dis. 2008;67(6):877-9.

34. Timpson N, Nordestgaard B, Harbord R, Zacho J, Frayling T, Tybjaerg-Hansen A, et al. C-reactive protein levels and body mass index: elucidating direction of causation through reciprocal Mendelian randomization. Int J Obes (Lond). 2011;35(2): $300-8$.

35. Halder I, Marsland A, Cheong J, Muldoon M, Ferrell R, Manuck $\mathrm{S}$. Polymorphisms in the CRP gene moderate an association between depressive symptoms and circulating levels of C-reactive protein. Brain Behav Immun. 2010;24(1):160-7.

36. Almeida O, Norman P, Allcock R, van Bockxmeer F, Hankey G, Jamrozik K, et al. Polymorphisms of the CRP gene inhibit inflammatory response and increase susceptibility to depression: the health in men study. Int J Epidemiol. 2009;38(4):1049-59.

37. Feskens E, Bloemberg B, Pijls L, Kromhout D. A longitudinal study on elderly men: the Zutphen Study. In: Schroots JJF, editor. Aging, Health and Competence. Amsterdam: Elsevier Science; 1993.

38. Schoenmaker M, de Craen AJ, de Meijer PH, Beekman M, Blauw GJ, Slagboom PE, et al. Evidence of genetic enrichment for exceptional survival using a family approach: the Leiden Longevity Study. Eur J Hum Genet. 2006;14(1):79-84.

39. van den Hombergh CE, Schouten EG, van Staveren WA, van Amelsvoort LG, Kok FJ. Physical activities of noninstitutionalized Dutch elderly and characteristics of inactive elderly. Med Sci Sports Exerc. 1995;27(3):334-9.

40. Giltay E, Geleijnse J, Zitman F, Hoekstra T, Schouten E. Dispositional optimism and all-cause and cardiovascular mortality in a prospective cohort of elderly dutch men and women. Arch Gen Psychiatry. 2004;61(11):1126-35.

41. Tempelman C (1987) Well-being in the elderly: development of the Scale Subjective Well-being in Older persons. University of Groningen. 
42. Sweat V, Starr V, Bruehl H, Arentoft A, Tirsi A, Javier E, et al. C-reactive protein is linked to lower cognitive performance in overweight and obese women. Inflammation. 2008;31(3):198-207.

43. Brull D, Serrano N, Zito F, Jones L, Montgomery H, Rumley A, et al. Human CRP gene polymorphism influences CRP levels: implications for the prediction and pathogenesis of coronary heart disease. Arterioscler Thromb Vasc Biol. 2003;23(11):2063-9.

44. Russell A, Cunninghame Graham D, Shepherd C, Roberton C, Whittaker J, Meeks J, et al. Polymorphism at the C-reactive protein locus influences gene expression and predisposes to systemic lupus erythematosus. Hum Mol Genet. 2004;13(1):137-47.

45. Steptoe A, O'Donnell K, Badrick E, Kumari M, Marmot M. Neuroendocrine and inflammatory factors associated with positive affect in healthy men and women: the Whitehall II study. Am J Epidemiol. 2008;167(1):96-102.

46. Fried S, Bunkin D, Greenberg A. Omental and subcutaneous adipose tissues of obese subjects release interleukin-6: depot difference and regulation by glucocorticoid. J Clin Endocrinol Metab. 1998;83(3):847-50.

47. Bastard J, Jardel C, Delattre J, Hainque B, Bruckert E, Oberlin F. Evidence for a link between adipose tissue interleukin- 6 content and serum C-reactive protein concentrations in obese subjects. Circulation. 1999;99(16):2221-2.

48. Giltay E, Geleijnse J, Zitman F, Buijsse B, Kromhout D. Lifestyle and dietary correlates of dispositional optimism in men: the Zutphen Elderly Study. J Psychosom Res. 2007;63(5):483-90.

49. Hayley S, Poulter M, Merali Z, Anisman H. The pathogenesis of clinical depression: stressor- and cytokine-induced alterations of neuroplasticity. Neuroscience. 2005;135(3):659-78.

50. Costanzo E, Lutgendorf S, Kohut M, Nisly N, Rozeboom K, Spooner S, et al. Mood and cytokine response to influenza virus in older adults. J Gerontol A Biol Sci Med Sci. 2004;59(12): 1328-33. 